\title{
Genistein Inhibits Proliferation of BRCA1 Mutated Breast Cancer Cells: The GPR30-Akt Axis as a Potential Target
}

\author{
Ga Yun Kim ${ }^{1,2}$, Jinyoung Suh ${ }^{2}$, Jeong-Hoon Jang ${ }^{2}$, Do-Hee Kim ${ }^{1,2}$, Ock Jin Park ${ }^{3}$, Sue K. Park ${ }^{4,5,6}$, Young-Joon Surh ${ }^{1,2,6}$ \\ 1 Department of Molecular Medicine and Biopharmaceutical Sciences, Graduate School of Convergence Science and Technology, Seoul National \\ University, ${ }^{2}$ Tumor Microenvironment Global Core Research Center, College of Pharmacy, Seoul National University, Seoul, ${ }^{3}$ Department of Food \\ and Nutrition, Hannam University, Daejeon, ${ }^{4}$ Department of Preventive Medicine, Seoul National University College of Medicine, ${ }^{5}$ Department \\ of Biomedical Science, Seoul National University Graduate School, ${ }^{6}$ Cancer Research Institute, Seoul National University, Seoul, Korea
}

\begin{abstract}
Background: BRCA1 mutated breast cancer cells exhibit the elevated cell proliferation and the higher metastatic potential. $G$ protein-coupled receptor 30 (GPR30) has been shown to regulate growth of hormonally responsive cancers, such as ovarian and breast cancers, and high expression of GPR30 is found in estrogen receptor (ER)-negative breast cancer cells. ER-negative breast cancer patients often have a mutation in the tumor suppressor gene, BRCA1. This study explored antiproliferative effects of genistein, a chemopreventive isoflavone present in legumes, and underlying molecular mechanisms in triple negative breast cancer cells with or without functionally active BRCA1.

Methods: Expression of BRCA1, GPR30 and Nrf2 was measured by Western blot analysis. Reactive oxygen species (ROS) accumulation was monitored by using the fluorescence-generating probe, $2^{\prime}, 7^{\prime}$-dichlorofluorescein diacetate. The effects of genistein on breast cancer cell viability and proliferation were assessed by the MTT, migration and clonogenic assays.

Results: The expression of GPR30 was dramatically elevated at both transcriptional and translational levels in BRCA1 mutated breast cancer cells compared to cells with wild-type BRCA1. Notably, there was diminished Akt phosporylation in GPR30 silenced cells. Treatment of BRCA1 silenced breast cancer cells with genistein resulted in the down-regulation of GPR30 expression and the inhibition of Akt phosphorylation as well as the reduced cell viability, migration and colony formation. Genistein caused cell cycle arrest at the $\mathrm{G}_{2} / \mathrm{M}$ phase in BRCA1-mutant cells through down-regulation of cyclin B1 expression. Furthermore, BRCA1-mutant breast cancer cells exhibited higher levels of intracellular ROS than those in the wild-type cells. Genistein treatment lowered the ROS levels through up-regulation of Nrf2 expression.

Conclusions: Lack of functional BRCA1 activates GPR30 signaling, thereby stimulating Akt phosphorylation and cell proliferation. Genistein induces G2/M phase arrest by down-regulating cyclin B1 expression, which is attributable to its suppression of GPR30 activation and Akt phosphorylation in BRCA1 impaired breast cancer cells.
\end{abstract}

(J Cancer Prev 2019;24:197-207)

Key Words: BRCA1, GPR30, Genistein, Akt, Breast cancer

\section{INTRODUCTION}

Breast cancer is one of the most frequently diagnosed malignancies in women, and especially the triple negative breast cancer (TNBC) type has a significantly worse prognosis as well as a higher recurrence rate [1]. The main reasons for the poor prognosis include a higher rate of metastasis and resistance to chemotherapy and/or radiotherapy. TNBC is characterized by lack of all three genes encoding estrogen receptor (ER), progesterone receptor and Her2/neu, and often associated with BRCA1 mutation [2].

$B R C A 1$ is one of the most commonly mutated tumor suppressor genes that accounts for a large proportion of hereditary breast cancer [3,4]. In addition, lack of the expression of functional

Received December 2, 2019, Revised December 16, 2019, Accepted December 16, 2019

Correspondence to: Young-Joon Surh

E-mail: surh@snu.ac.kr, ORCID: Young-Joon Surh, https://orcid.org/0000-0001-8310-1795

Copyright (C) 2019 Korean Society of Cancer Prevention

(c) This is an Open Access article distributed under the terms of the Creative Commons Attribution Non-Commercial License (http://creativecommons.org/licenses/by-nc/4.0) which permits unrestricted non-commercial use, distribution, and reproduction in any medium, provided the original work is properly cited. 
BRCA1 is observed in many cases of sporadic breast and ovarian cancers, signifying the absence or mutation of BRCA1 as an essential causative factor in these malignancies [4,5]. When $B R C A 1$ is mutated, damaged DNA cannot be repaired correctly, which causes genetic alterations, leading to genomic instability, and eventually malignant transformation of cells. Moreover, loss of BRCA1 accounts for invasion and metastasis of breast cancer cells [6-8].

A seven-transmembrane receptor $\mathrm{G}$ protein-coupled receptor 30 (GPR30) has been reported to mediate rapid non-genomic signals of estrogens $[9,10]$. The stimulation of GPR30 in ERnegative breast cancer cells can result in the reduced cell proliferation and increased apoptosis. GPR30 has been suggested to activate Akt signals involved in cancer cell proliferation and cell cycle progression [9,11,12]. Further, a positive correlation between expression of GPR30 and a reactive oxygen species (ROS) level has been reported [11,13]. The increased ROS production is often found in cancer cells, which is strongly associated with decreased activation of Nrf2, a key transcription factor involved in regulation of antioxidant gene expression [14].

Epidemiological studies have demonstrated that consumption of soy foods lowers the risk of breast cancer [15,16]. Genistein, one of the most abundant isoflavones present in soybeans, has a chemopreventive effect on mammary carcinogenesis [17-19]. The chemopreventive and anticarcinogenic activities of genistein have been attributed, at least in part, to its capability to antagonize the ER. However, its effect on survival and proliferation of estrogen negative cells, especially differing by the presence or absence of BRCA1 has not been fully clarified. Genistein has been reported to block $\mathrm{G}_{2} / \mathrm{M}$ progression of the cancer cell cycle [20,21]. There have been investigations that explore the relationship between inhibition of Akt signaling and $\mathrm{G}_{2} / \mathrm{M}$ arrest [22]. Overexpression of cyclin B1, a key player of $\mathrm{G}_{2} / \mathrm{M}$ phase cell cycle machinery, is associated with the hyperactivation of Akt signaling [23].

This study aimed to examine the effects of genistein on growth of TNBC cells with impaired BRCA function. We have found that genistein suppresses TNBC proliferation most likely through inactivation of the GPR30-Akt signaling.

\section{MATERIALS AND METHODS}

\section{Materials}

Genistein was purchased from Sigma-Aldrich (St. Louis, MO, USA). Dulbecco's modified Eagle's Medium (DMEM) and Rosewell Park Memorial Institute (RPMI) 1640 medium were obtained from Gibco BRL (Grand Island, NY, USA). FBS was supplied from GenDEPOT (Barker, TX, USA). TRIzol ${ }^{\circledR}$, SYBR $^{\circledR}$ safe DNA gel stain and Lipofectamine ${ }^{\circledR}$ RNAiMAX were purchased from Invitrogen (Carlsbad, CA, USA). Primary antibodies against BRCA1, P-Akt and Akt were obtained from Cell Signaling Technology (Danvers, MA, USA). Primary antibodies against cyclin B1 and actin were products of Santa Cruz Biotechnology (Santa Cruz, CA, USA). GPR30 and Nrf2 primary antibodies were supplied from Abcam (Cambridge, MA, USA). Anti-rabbit and anti-mouse secondary antibodies were purchased from Invitrogen (Carlsbad, CA, USA).

\section{Cell culture}

MDA-MB-231 and HCC1937 cells were cultured in DMEM and RPMI, respectively. Each medium was supplemented with $10 \%$ FBS and $1 \%$ antibiotic-antimycotic. The cells were maintained at $37^{\circ} \mathrm{C}$ with humidified atmosphere of $5 \% \mathrm{CO}_{2}$ and $95 \%$ air.

\section{MTT assay}

MDA-MB-231 and HCC1937 cells were counted and seeded at a density of $1.6 \times 10^{4}$ per well in 48 -well plates. After 24 hours of incubation, the cells were treated with various concentrations of genistein $(10,25,50$, or $100 \mu \mathrm{M})$. Cell viability was measured at 72 hours. Thiazolyl blue tetrazolium bromide (Sigma-Aldrich, St. Louis, MO, USA) was added at a concentration of $0.5 \mathrm{mg} / \mathrm{mL}$. After 3 hours of incubation, dimethyl sulfoxide was added to solubilize the formazan crystals formed. The absorbance was measured at $570 \mathrm{~nm}$ using a micro-plate reader (Bio-Rad Laboratories, Hercules, California, USA).

\section{Migration assay}

Two-well Culture-Inserts (Ibidi ${ }^{\circledR}$ ) were attached to 12-well plates. MDA-MB-231 and HCC1937 cells were seeded at a density of $1.5 \times 10^{4}$ for MDA-MB-231 and $2 \times 10^{4}$ for HCC1937 cells per each well in the inserts. After 24 hours of incubation, the silicon inserts were removed, and $50 \mu \mathrm{M}$ genistein was added. After incubation for another 24 hours, the cells were photographed under a microscope. The procedure was repeated using BRCA1siRNA transfected MDA-MB-231 cells.

\section{Clonogenic assay}

MDA-MB-231 and HCC1937 cells were plated in 6-well plates at a density of 300 cells per well. Medium was changed every other day. After 7 days of incubation, genistein $(10$ or $50 \mu \mathrm{M})$ was added. Cells were further cultured for 7 days. The cells were then washed with PBS and fixed in cold methanol for 10 minutes. The colonies 
were stained using $0.5 \%$ crystal violet and imaged by the LAS-4000 image reader (Fuji Film, Tokyo, Japan).

\section{Transient transfection with siRNA}

MDA-MB-231 cells were reverse transfected with BRCA1siRNA, and HCC1937 cells were reverse transfected with GPR30siRNA. The target sequences used for transfection are as follows. For BRCA1-siRNA, 5'-CUA GAA AUC UGU UGC UAU G-3' (sense), and 5'-C AUA GCA ACA GAU UUC UAG-3' (antisense). For GPR30-siRNA, 5'-ACA ACU GCG GUG AUG AUG U-3' (sense), and 5'-ACA UCA UCA CCG CAG UUG U-3'(antisense). Each siRNA (20 $\mu \mathrm{M}$ ) was diluted in Opti-MEM to make the final volume of $20 \mathrm{nM}$. Lipofectamine $^{\circledR}$ RNAiMAX reagent diluted in Opti-MEM was then added, and the mixture was incubated for 15 minutes and added to newly seeded cells. After 48 hours incubation, cells were harvested for further experiments.

\section{Western blot analysis}

Cells were harvested at designated time points. Cells were washed using cold PBS and collected as pellets. Cell pellets were suspended in $1 \times$ cell lysis buffer ( $20 \mathrm{mM}$ Tris- $\mathrm{HCl}$ [pH 7.5], 150 $\mathrm{mM} \mathrm{NaCl}, 1 \mathrm{mM} \mathrm{Na}$ EDTA, $1 \mathrm{mM}$ EGTA, 1\% Triton, $2.5 \mathrm{mM}$ sodium pyrophosphate, $1 \mathrm{mM}$ glycerophosphate, $1 \mathrm{mM} \mathrm{Na}_{3} \mathrm{VO}_{4}$, $1 \mu \mathrm{g} / \mathrm{mL}$ leupeptin; Cell Signaling Technology) supplemented with a protease inhibitor and 0.1 mM PMSF. After incubation in ice for 1 hour, the mixture was centrifuged at $13,000 \times g$ for 15 minutes at $4^{\circ} \mathrm{C}$. Supernatant was collected as a whole cell lysate. Protein concentrations were determined by the bicinchoninic acid assay. Protein samples were made by mixing equal amounts of protein with the SDS loading dye and boiled at $99^{\circ} \mathrm{C}$ for 5 minutes. Proteins samples were electrophoresed in SDS-PAGE and were transferred to polyvinylidene fluoride membranes (German Laboratory, Ann Arbor, MI, USA). The blots were blocked with $5 \%$ fat-free dry milk in TBS buffer containing $0.1 \%$ Tween-20 (TBST) for 1 hour at room temperature. The blots were then incubated using an indicated primary antibody overnight at $4^{\circ} \mathrm{C}$. The membranes were washed with TBST and incubated with respective horseradish peroxidase conjugated secondary antibodies for 1 hour. After incubation, the blots were washed again with TBST and was visualized with the enhanced chemiluminescence substrate detection reagent using the LAS-4000 image reader.

\section{Reverse transcription-PCR}

Total RNA was isolated from MDA-MB-231 and HCC1937 cells by using TRIzol ${ }^{\mathbb{R}}$ (Invitrogen) according to the manufacturer's protocol. To generate cDNA, $1 \mu \mathrm{g}$ of total RNA was reverse transcribed with moloney murine leukemia virus reverse transcriptase (Promega, Madison, WI, USA) at $42^{\circ} \mathrm{C}$ for $50 \mathrm{~min}$ and $72^{\circ} \mathrm{C}$ for $15 \mathrm{~min}$. One $\mu \mathrm{l}$ of cDNA was amplified in sequential reactions using Solg ${ }^{\mathrm{TM}} 2 \times$ Taq PCR Smart mix 1 (SolGent, Seoul, Korea). The primer sequence and conditions used for each PCR reactions are as follows; GPR30, 5'-AGTCGGATGTGAGGTTCAG-3' and 5'- TCTGTGTGAGGAGTGCAAG-3', 55 C. Amplification products were resolved by $2 \%$ agarose gel electrophoresis, stained with SYBR green and visualized by the LAS-4000 image reader.

\section{Measurement of reactive oxygen species}

To measure the intracellular accumulation of ROS, a fluorescent probe 2,7-dichlorofluoresecein diacetate (DCF-DA) was used. MDA-MB-231 and HCC1937 cells were rinsed with Hank's balanced salt solution (HBSS), and $10 \mu \mathrm{M}$ of DCF-DA was loaded. After 30 minutes incubation at $37^{\circ} \mathrm{C}$, cells were visualized under a live cell image microscope.

\section{Cell cycle analysis}

MDA-MB-231 (scrambled siRNA and BRCA1-siRNA transfected) and HCC1937 cells were treated with genistein for 72 hours. The cells were collected, washed with PBS and fixed using $70 \%$ ethanol for 2 hours at $4^{\circ} \mathrm{C}$. Then, the cells were washed with HBSS, added with RNase and stained with propidium iodide. The proportion of cells in each phase of cell cycle was measured using BD FACSCalibur (Becton Dickinson Biosciences).

\section{Xenograft assay}

Animal experiments were conducted in accordance with institutional guidelines for the care and use of experimental animals. Each side of a BALB/c nude mice was injected subcutaneously with $1 \times 10^{7}$ control or BRCA1-siRNA transfected MDA-MB-231 cells. After one month of inoculation, the size of tumors was measured. The formula used for calculation of tumor volume was $0.5 \times$ larger diameter $\times(\text { smaller diameter })^{2}$.

\section{Statistical analysis}

Results were expressed as the means of \pm SD of three independent experiments. The statistical significance of the difference between groups was determined using Student's $t$ test. Analysis was performed using SigmaPlot 8.0. $P<0.05$ was considered a statistically significant difference. 


\section{RESULTS}

\section{Effects of BRCA1 impairment on proliferation of triple negative breast cancer cells}

BRCA1 impaired cells are known to proliferate aggressively [24]. In agreement with this notion, HCC1937 cells harboring mutated $B R C A 1$ showed significantly increased phosphorylation of Akt and expression of GPR30 compared to MDA-MB-231 cells with wild-type BRCAI (Fig. 1A). The level of the mRNA transcript of GPR30 was also markedly elevated in HCC1937 cells (Fig. 1B). In addition, the protein expression of GPR30 was significantly elevated in BRCA1-siRNA transfected MDA-MB-231 cells (Fig. 1C). Silencing of GPR30in HCC1937 cells reduced the P-Akt level (Fig. 1D), suggesting that GPR30 activates Akt signaling.
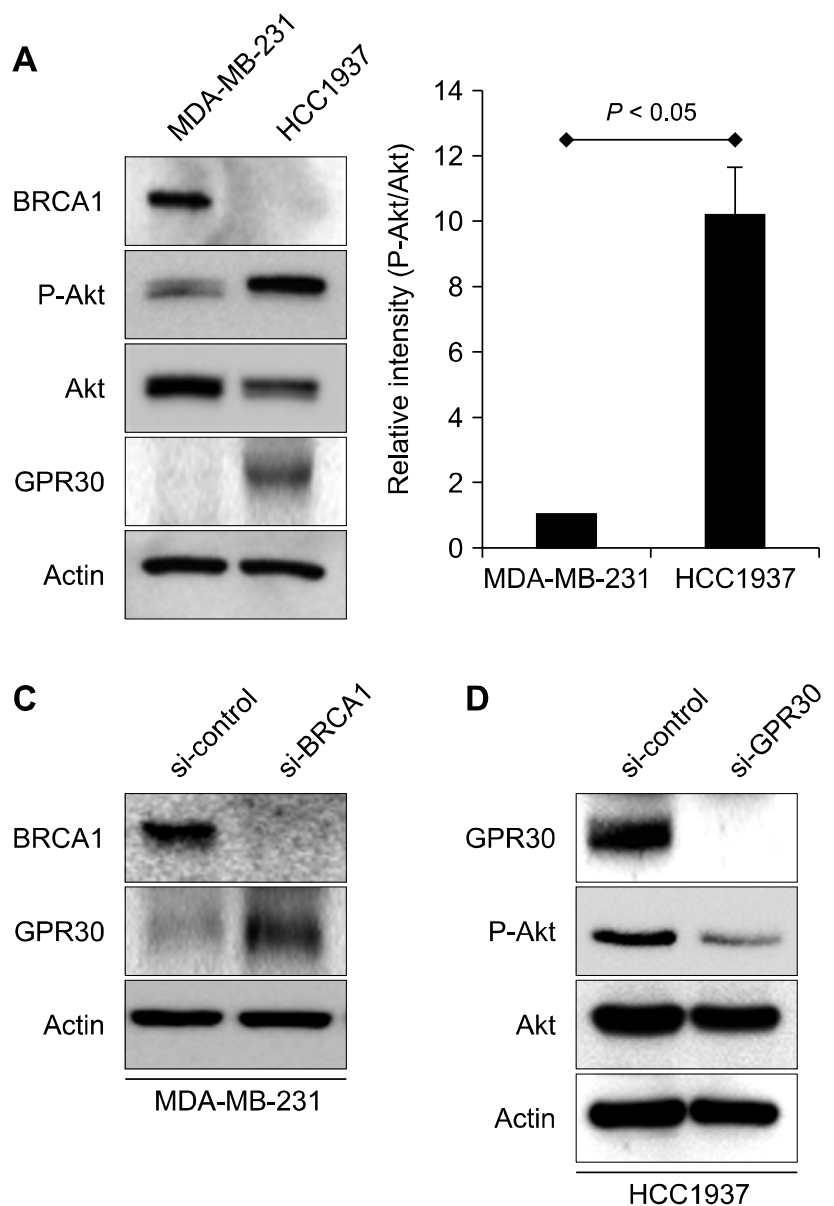

2. Effects of genistein on the viability of triple negative breast cancer cells

Next, MDA-MB-231 and HCC1937 cells were compared with regard to their susceptibility to the cytotoxic effect of genistein. For both cell lines, treatment with genistein lowered the cell viability in a concentration-dependent manner (Fig. 2A). Compared to cells with wild-type $B R C A 1$, TNBC cells with mutated (Fig. 2A) or silenced (Fig. 2B) BRCA1 were apprenetly more sensitive to genistein.

3. Effects of genistein on the migration of triple negative breast cancer cells and their colony formation

The anti-proliferative effect exerted by genistein was extended
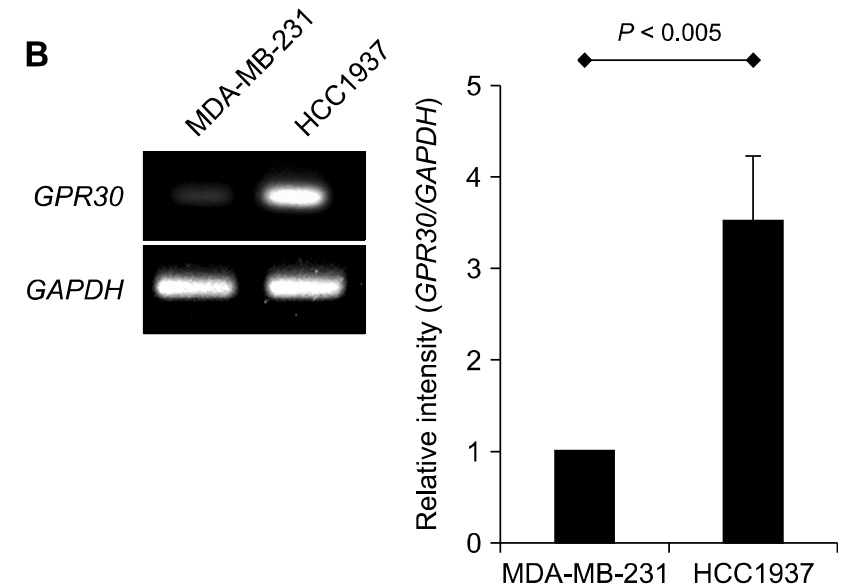

Figure 1. Elevated Akt phosphorylation and GPR30 expression in BRCA1 deficient triple negative breast cancer cells. (A) Phosphorylation of Akt and GPR30 expression were compared with respect to the BRCA1 status in MDA-MB-231 and HCC1937 cells by Western blot analysis. (B) The levels of GPR30 mRNA were measured by reverse transcription-PCR. (C) MDA-MB-231 cells were transfected with BRCA1-siRNA, and then expression of BRCA1 and GPR30 was determined by immunoblot analysis. (D) HCC1937 cells are reverse transfected with $20 \mathrm{nM}$ GPR30-siRNA. After silencing GPR30 in HCC1937 cells, P-Akt expression level was measured by Western blot analysis. GAPDH, glyceraldehyde 3-phosphate dehydrogenase. 

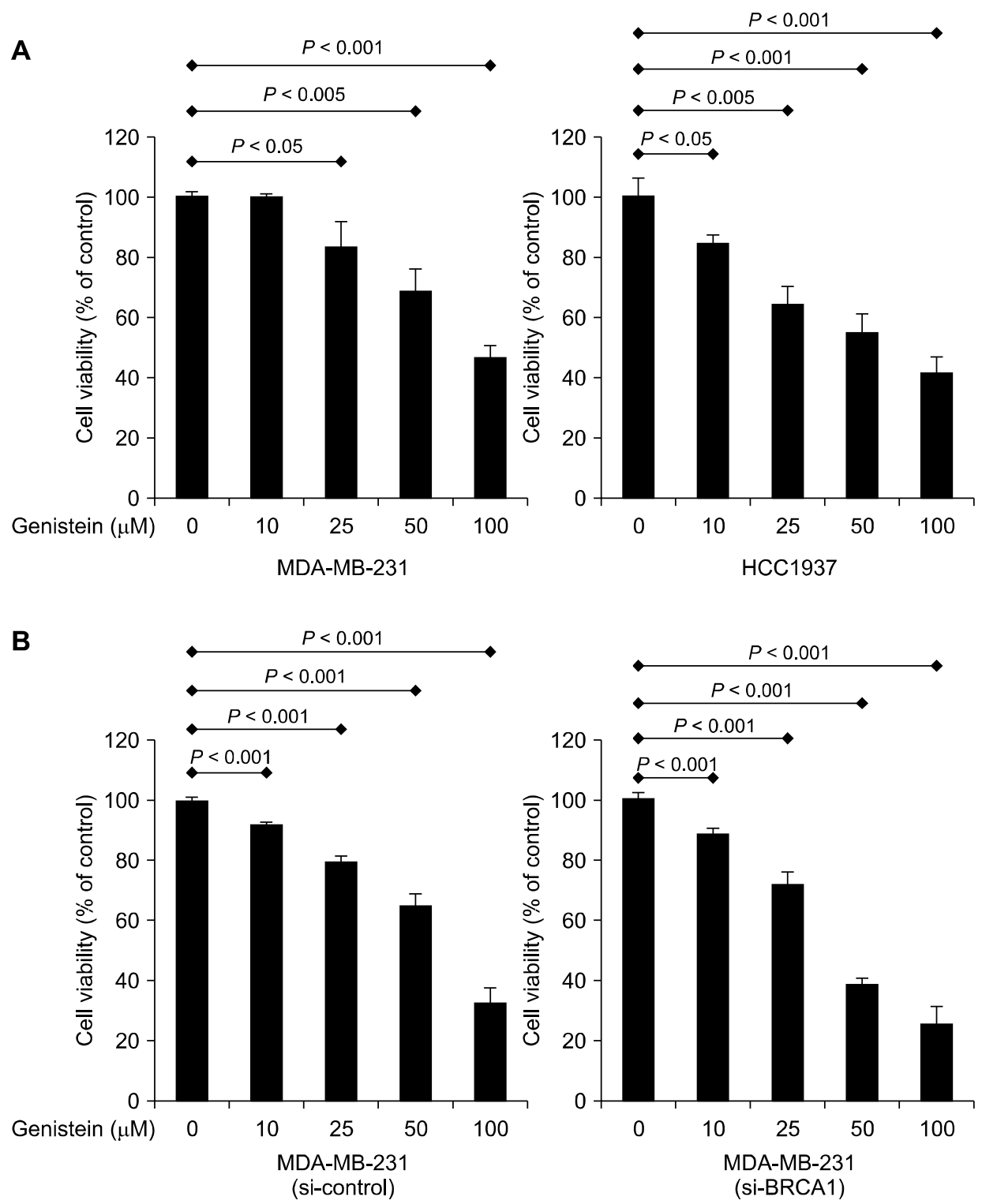

Figure 2. Effects of genistein on viability of MDA-MB-231 and HCC1937 cells. Cell viability was assessed by the MTT assay. (A) MDA-MB-231 and HCC1937 cells were seeded into 48-well-plates at a density of $1.6 \times$ $10^{4}$ cells per well. After 24 hours of incubation, the cells were exposed to increasing concentrations $(10,25,50$, or $100 \mu \mathrm{M}$ ) of genistein for 72 hours. Then, the cells were incubated with $0.5 \mathrm{mg} / \mathrm{mL}$ of MTT in media for additional 3 hours, and the absorbance was measured at $570 \mathrm{~nm}$. (B) The same procedure was repeated with scrambled siRNA in BRCAl-siRNA transfected MDA-MB-231 cells.

to its capability to inhibit cell migration and colony formation. When treated with genistein for 24 hours, both MDA-MB-231 and HCC1937 cells showed reduced migration, which was more pronounced in HCC1937 cells (Fig. 3A). Likewise, MDA-MB-231 cells transfected with $B R C A 1$-siRNA exhibited greater reduction in migration upon treatment with genistein (Fig. 3B). In addition, genistein treatment markedly decreased the number and the size of colonies (Fig. 3C). Again, BRCA1 impaired TNBC cells were more responsive to genistein compared to those with functionally active BRCA1.
4. Comparative effects of genistein on cell cycle progression in MDA-MB-231 cells with and without $B R C A 1$ silencing

To determine whether genistein could regulate cell cycle machinery in its anti-proliferative activity, the cell cycle distribution was measured by flow cytometry. The proportion of cells in the G2/M phase increased 14\% in BRCA1-siRNA transfected cells (Fig. 4A). As GPR30 is known to activate Akt [9,10,25], which is an upstream kinase of cyclin B1 [23], expression of these two signaling molecules upon genistein treatment was examined. Genistein treatment to MDA-MB-231 cells transfected with control siRNA did not cause any changes in the expression level of cyclin B1. On the other hand, BRCA1-siRNA transfected 

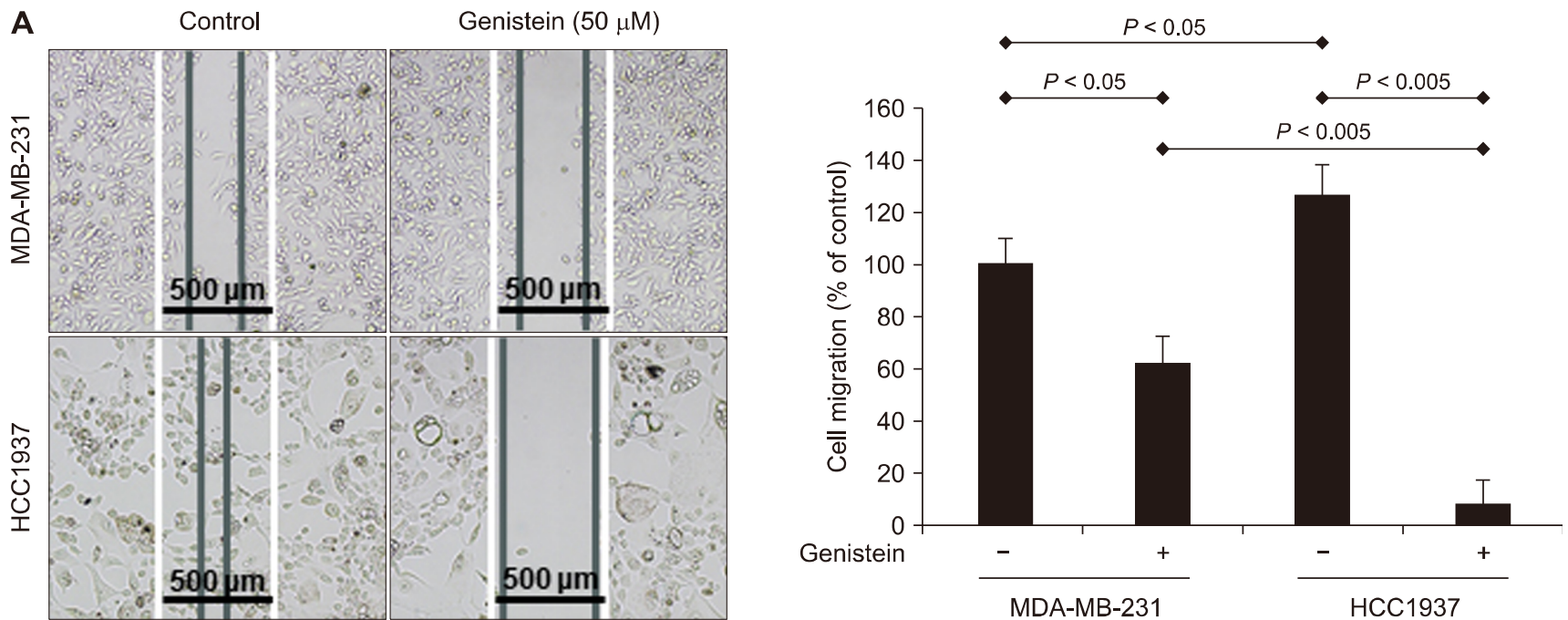

B

MDA-MB-231
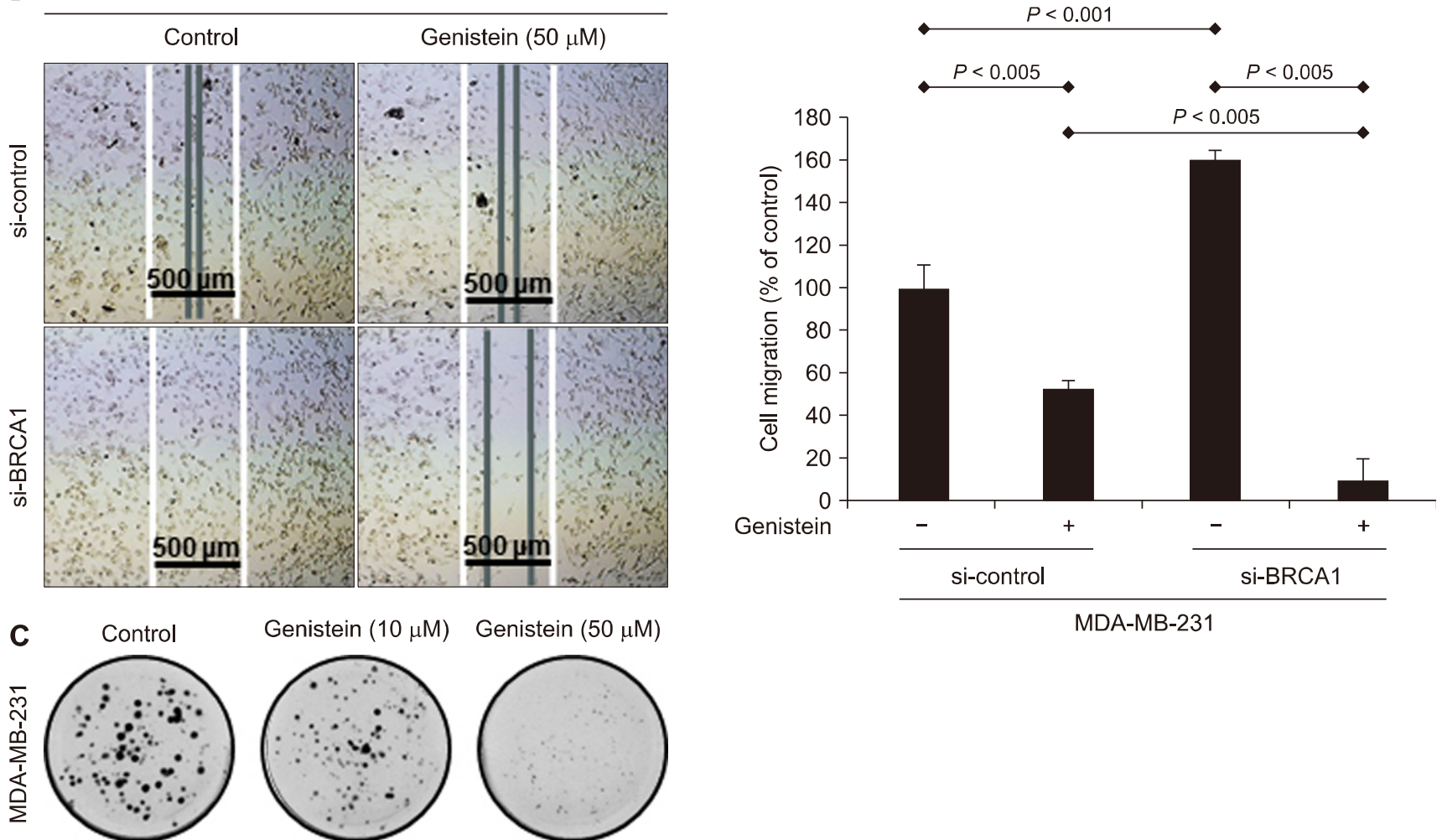

Genistein $(10 \mu \mathrm{M})$

Genistein $(50 \mu \mathrm{M})$

MDA-MB-231
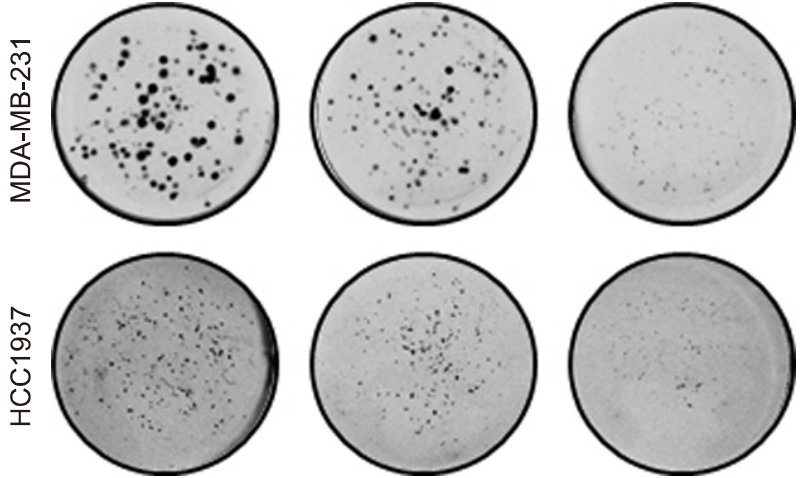

Figure 3. Inhibitory effects of genistein on migration and colony formation of MDA-MB-231 and HCC1937 cells. (A) The cells were seeded into two chambers of an insert $\left(1.5 \times 10^{4}\right.$ per well for MDA-MB-231 and $2 \times 10^{4}$ per well for HCC1937 cells). After 24 hours of incubation, the insert was removed, and the cells were treated with $50 \mu \mathrm{M}$ genistein. After additional 24 hours of incubation, the gap was measured under a microscope. (B) The same procedure was repeated for scrambled siRNA and BRCA1-siRNA transfected MDA-MB-231 cells. (C) Cells were seeded in a 6-well plate at a density of $5 \times 10^{2}$ cells per well. The cells were then incubated for a week followed by treatment with 10 or $50 \mu \mathrm{M}$ genistein. After additional one week of incubation, the cells were stained with crystal violet for visualization under a microscope. 
A

MDA-MB-231
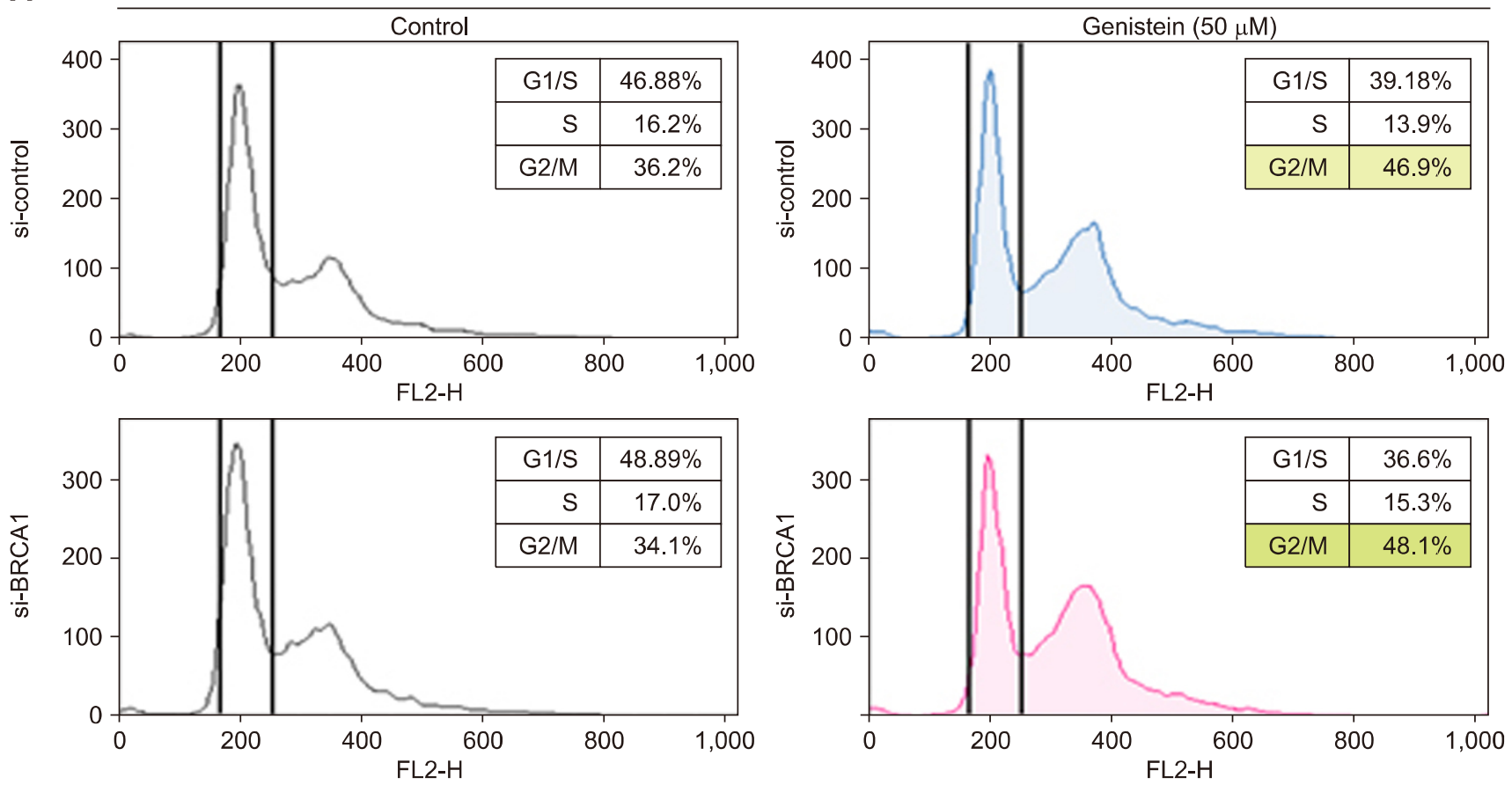

B

MDA-MB-231

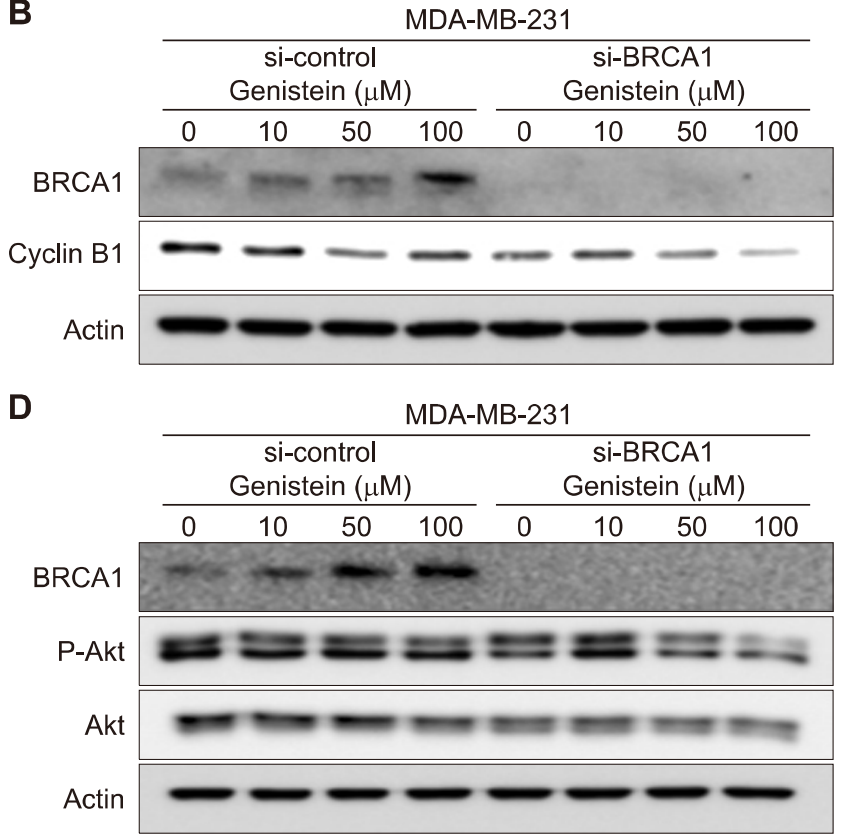

C

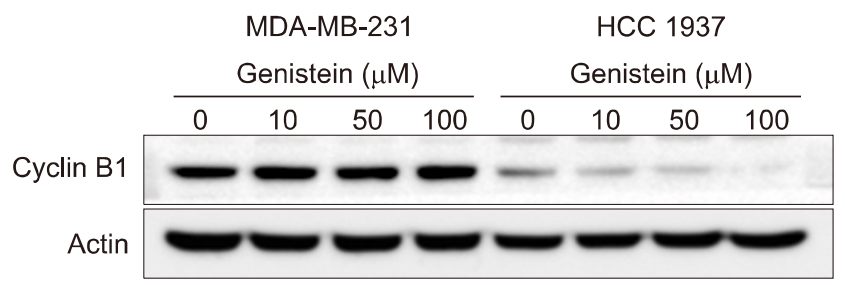

Figure 4. Involvement of genistein in the cell cycle arrest at the G2/M phase. (A) MDA-MB-231 cells were transfected with either scrambled siRNA or BRCA1-siRNA, and the effects of genistein on cell cycle progression were assessed. MDA-MB-231 cells were treated with $50 \mu \mathrm{M}$ genistein for 72 hours. Harvested cells were washed using PBS and fixed with ice-cold $70 \%$ ethanol for 2 hours at $4^{\circ} \mathrm{C}$. Fixed cells were washed with HBSS, followed by staining with propidium iodide. Cell population was measured by FACS analysis. (B) The experiment was repeated using MDA-MB-231 cells with and without BRCA1-siRNA knockdown to compare the effect of genistein on cyclin B1 expression levels. (C) The effects of genestein on cyclin B1 expression was compared in both MDA-MB-231 and HCC1937 cells. (D) MDA-MB-231 cells were transfected with either scrambled siRNA or BRCA1-siRNA, and then treated with genistein. Expression and phosphorylation levels of Akt were determined by Western blot analysis. 
cells showed a decrease in the expression of cyclin B1 upon genistein treatment (Fig. 4B). Likewise, genistein inhibited cyclin B1 expression in HCC1937 cells harbouring mutant BRCA1, but not in MDA-MB-231 cells with functionally active BRCA1 gene (Fig. 4C). In addition, there was more pronounced reduction in phosphorylation of Akt in BRCA1-siRNA transfected MDA-MB231 cells (Fig. 4D).

\section{Effects of genistein on $\mathrm{G}$ protein-coupled receptor} 30 expression and reactive oxygen species accumulation in triple negative breast cancer cells with BRCA1 impairment

While expression of GPR30 was decreased in HCC1937 cells by genistein treatment, such change was not observed in MDA-MB-231 cells that barely express GPR30(Fig. 5A). Next, the intracellular ROS level was measured upon genistein treatment. HCC1937 cells exhibited higher intracellular levels of ROS than did the MDA-MB-231 cells (Fig. 5B). While both MDA-MB-231 and HCC1937 cells treated with genistein $(50 \mu \mathrm{M})$ showed a diminished ROS level, the decrease was more noticeable in HCC1937 cells (Fig. 5B). In parallel with reduction in intracellular ROS accumulation, there was a concomitant increase in the Nrf2 protein level following genistein treatment (Fig. 5C). These results suggest that $\mathrm{Nrf} 2$ activation by genistein may account for its inhibition of intracellular ROS accumulation in HCC1937 cells.

\section{Effects of BRCA1 silencing on growth of triple negative breast cancer cells in athymic nude mice} Association between BRCA1 and tumorigenesis has been
A

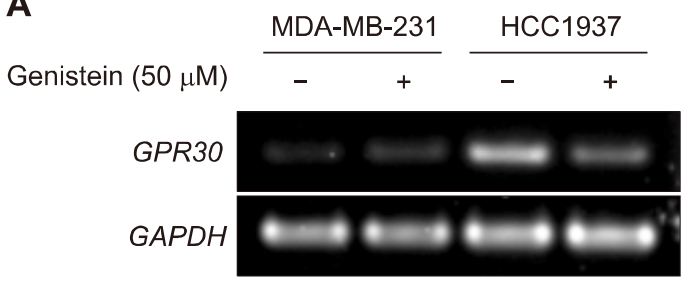

B

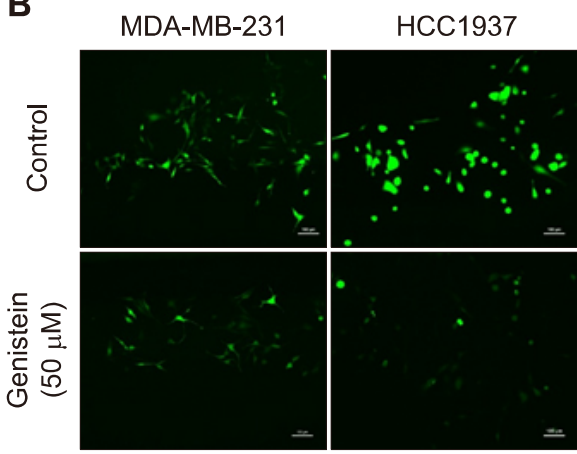

C

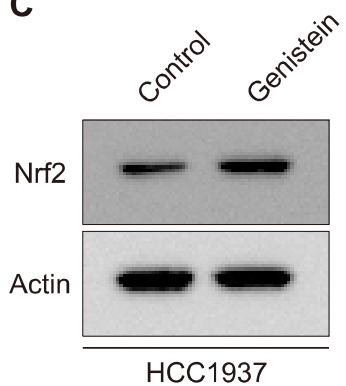

Figure 5. Effects of genistein on GPR30 expression and reactive oxygen species (ROS) generation. (A) MDA-MB-231 and HCC1937 cells were treated with $50 \mu \mathrm{M}$ Genistein. After 48 hours of incubation, the mRNA expression of GPR30 was assessed by reverse transcription-PCR. (B) The intracellular ROS levels in genistein treated MDA-MB-231 and HCC1937 cells were measured using a DCF-DA fluorescent dye. Cells were exposed to either dimethyl sulfoxide or $50 \mu \mathrm{M}$ genistein for 24 hours. Images of cellular fluorescence were acquired by using a live cell image microscope. (C) The protein level of Nrf2 in HCC1937 with and without genistein treatment for 72 hours was measured by Western blot analysis. GAPDH, glyceraldehyde 3-phosphate dehydrogenase.
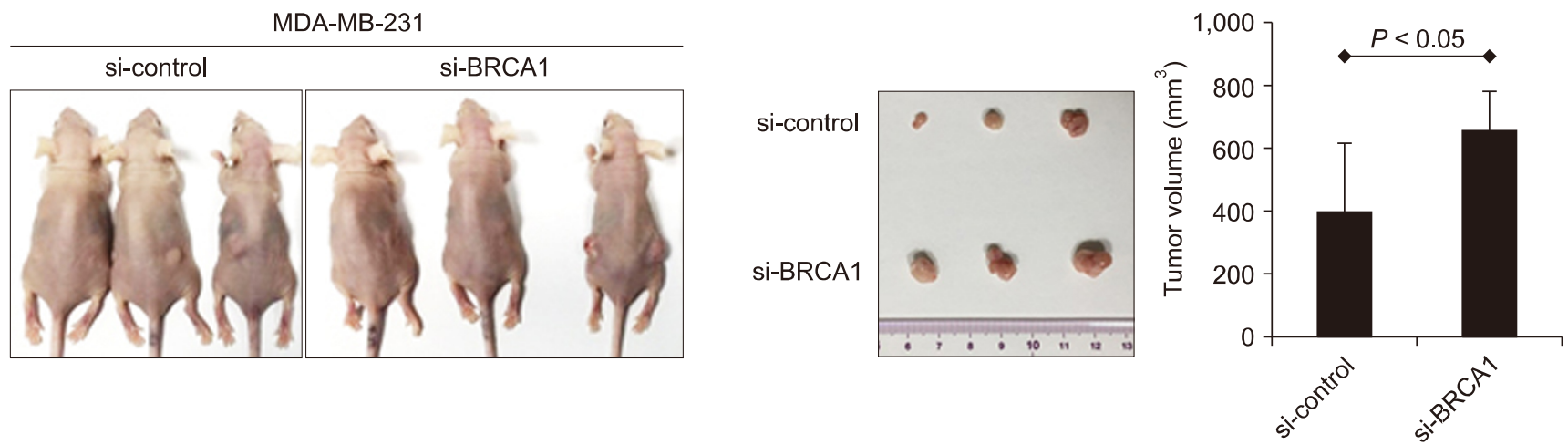

Figure 6. Effects of BRCA1 gene status on xenograft tumor formation. Control and BRCA1 knockdown MDA-MB-231 cells were prepared, and $1 \times 10^{7}$ cells were injected to each side of BALB/C mouse. The mice were sacrificed after one month, and the tumor volume was measured with digital calipers. The calculated formula is $0.5 \times$ larger diameter $\times$ (smaller diameter) $)^{2}$. 
extensively investigated [26,27]. To determine whether BRCA1 deficiency has an effect on tumor forming capability in vivo, control and BRCA1 knockdown MDA-MB-231 cells were injected into $\mathrm{BALB} / \mathrm{C}$ nude mice at a density of $1 \times 10^{7}$ cells per side. After one month of inoculation, the size of tumors was measured. As shown in Figure 6, tumors derived from BRCA1 knockdown cells had significantly larger volume compared to those from the cells with wild-type BRCA1. It'll be necessary to determine the effects of genistein on growth of BRCA1 silenced MDA-MB-231 cells in a xenograft model.

\section{DISCUSSION}

The present study demonstrates that genistein inhibits the proliferation and growth of TNBC cells with mutant BRCA1 by targeting GPR30. This led to the reduced expression of Akt, and subsequently cyclin B1, a key enzyme involved in cell cycle progression in the $\mathrm{G}_{2} / \mathrm{M}$ phase. In addition, genistein suppressed the overproduction of ROS in BRCA1 deficient cells, apprently through activation of Nrf2 signaling.

Estrogen plays a key role in the development and progression of breast cancers. Estrogen binds to the receptor to exert its (patho)physiologic function. Besides classical ER, such as ER $\alpha$ and ER $\beta$, GPR30 can also respond to estrogen. In contrast to ER $\alpha$ and ER $\beta$, GPR30 is located at the cell surface membrane for a rapid response to estrogen [28]. Breast cancers are classified ER positive, ER negative, and triple negative, depending on their receptor types. TNBC is the most common histological subtype associated with BRCA1 mutation [29]. Since prevalence of BRCA1 mutation in detrimental TNBC patients is relatively high, this prompted us to investigate the proliferation and progression of TNBC with BRCA1 mutation. For this purpose, a BRCA1 mutated HCC1937 cell line was selected. This cell line harbors 5382C insertion, the second most common $B R C A 1$ mutation. Besides BRCA1 mutated HCC1937 cells, BRCA1 silenced MDA-MB-231 cells were examined to further corroborate the findings on the role of BRCA1 in cell progression. Even though a substantial body of evidence suggests that BRCA1 deficient breast cancer is highly aggressive, the precise mechanisms by which BRCAl exerts its oncogenic functions have not been thoroughly scrutinized.

One of the salient features of our present study is demonstration of the possible involvement of GPR30 signaling in the aggressive growth and progression of breast cancer cells with BRCA1 impairment. The expression of GPR30 was dramatically elevated at both transcriptional and translational levels in BRCA1 mutant breast cancer cells in comparison to the wild-type cells. In
BRCA1 mutated HCC1937 cells, there was elevated expression of cell proliferation which was associated with enhanced Akt phosphorylation and GPR30 expression. When the tumor suppressor BRCA1 is not functioning properly, Akt is activated [30]. The elevated phosphorylation of Akt could be attributable to an increase in GPR30 because this receptor is reported to be associated with the activation of epidermal growth factor receptor-PI3K-Akt axis [31].

Genistein, a major phytochemical found in soy beans, has been extensively investigated for its chemoprevetive and anticarcinogenic potential [17-19]. In our present study, the effects of genistein on proliferation and progression of BRCA1 mutated TNBC cells were investigated. Genistein downregulated the expression of GPR30 and inhibited the phosphorylation of Akt, leading to the reduced expression of cyclin B1. This result suggests that BRCA1 deficiency/impairment may confer sensitivity to genistein. In support of this supposition, there is a report demonstrating the increased sensitivity to geinstein in breast cancer cells harboring mutant BRCA1 [32]. However, the exact molecular mechanism whereby genistein exerts anti-proliferative effects in breast cancer cells with an impaired BRCA1 function awaits further study.

It can be postulated that GPR30 might be a clue for differential effects of geinstein in breast cancer cells depending on the BRCA1 status. Highly expressed GPR30 and phosphorylated Akt in BRCA1 impaired breast cancer cells would render them more susceptible to genistein-induced growth suppression. Indeed, genistein exerts a stronger inhibitory effect on proliferation and/or migration in of BRCA1-mutant HCC1937 cells than in MDA-MB-231 cells with wild-type BRCA1. It was noticeable that BRCA1 impairment resulted in the overproduction of ROS. In line with this notion, Nrf2 in HCC1937 cells was barely detected. It would be worthwhile determining whether BRCA1 inhibits intracellular production of ROS and subsequently GPR30 signaling through activation of Nrf2.

In conclusion, this study provides evidence for the inhibitory effect of BRCA1 on aberrant proliferation, migration and progression of breast cancer cells. BRCA1 mutation leads to activation of the GPR30-Akt signaling, resulting in aggressiveness of TNBC cells. Further study is required to unravel whether geinstein acts in BRCA1 lacking cells by directly targeting GPR30.

\section{ACKNOWLEDGMENTS}

This work was supported by the Seoul National University Research Grant in 2016. 


\section{CONFLICTS OF INTEREST}

No potential conflicts of interest were disclosed.

\section{ORCID}

Ga Yun Kim,

https://orcid.org/0000-0002-7519-0414

Jinyoung Suh,

https://orcid.org/0000-0002-8878-9151

Jeong-Hoon Jang,

https://orcid.org/0000-0002-5925-6524

Do-Hee Kim,

https://orcid.org/0000-0002-9636-8293

Sue K. Park,

https://orcid.org/0000-0001-5002-9707

Young-Joon Surh,

https://orcid.org/0000-0001-8310-1795

\section{REFERENCES}

1. Torre LA, Bray F, Siegel RL, Ferlay J, Lortet-Tieulent J, Jemal A. Global cancer statistics, 2012. CA Cancer J Clin 2015;65:87-108.

2. Dent R, Trudeau M, Pritchard KI, Hanna WM, Kahn HK, Sawka CA, et al. Triple-negative breast cancer: clinical features and patterns of recurrence. Clin Cancer Res 2007;13(15 Pt 1):4429-34.

3. Antoniou AC, Pharoah PD, McMullan G, Day NE, Stratton MR, Peto J, et al. A comprehensive model for familial breast cancer incorporating BRCA1, BRCA2 and other genes. $\mathrm{Br} \mathrm{J}$ Cancer 2002;86:76-83.

4. Taylor J, Lymboura M, Pace PE, A'hern RP, Desai AJ, Shousha S, et al. An important role for BRCA1 in breast cancer progression is indicated by its loss in a large proportion of non-familial breast cancers. Int J Cancer 1998;79:334-42.

5. McCoy ML, Mueller CR, Roskelley CD. The role of the breast cancer susceptibility gene 1 (BRCA1) in sporadic epithelial ovarian cancer. Reprod Biol Endocrinol 2003;1:72.

6. Deng CX. BRCA1: cell cycle checkpoint, genetic instability, DNA damage response and cancer evolution. Nucleic Acids Res 2006:34:1416-26.

7. Zhang J, Powell SN. The role of the BRCA1 tumor suppressor in DNA double-strand break repair. Mol Cancer Res 2005;3:531-9.

8. Turner N, Tutt A, Ashworth A. Hallmarks of 'BRCAness' in sporadic cancers. Nat Rev Cancer 2004:4:814-9.

9. Ge X, Guo R, Qiao Y, Zhang Y, Lei J, Wang X, et al. The G protein-coupled receptor GPR30 mediates the nontranscriptional effect of estrogen on the activation of PI3K/Akt pathway in endometrial cancer cells. Int J Gynecol Cancer 2013;23:52-9.

10. Revankar CM, Cimino DF, Sklar LA, Arterburn JB, Prossnitz ER. A transmembrane intracellular estrogen receptor mediates rapid cell signaling. Science 2005:307:1625-30.

11. Lei B, Peng W, Xu G, Wu M, Wen Y, Xu J, et al. Activation of G protein-coupled receptor 30 by thiodiphenol promotes pro- liferation of estrogen receptor $\alpha$-positive breast cancer cells. Chemosphere 2017;169:204-11.

12. Zhang J, Yang Y, Zhang Z, He Y, Liu Z, Yu Y, et al. Gankyrin plays an essential role in estrogen-driven and GPR30-mediated endometrial carcinoma cell proliferation via the PTEN/PI3K/AKT signaling pathway. Cancer Lett 2013;339:279-87.

13. Prossnitz ER. GPER modulators: opportunity Nox on the heels of a class Akt. J Steroid Biochem Mol Biol 2018;176:73-81.

14. Kensler TW, Wakabayashi N. Nrf2: friend or foe for chemoprevention? Carcinogenesis 2010;31:90-9.

15. Lee SA, Shu XO, Li H, Yang G, Cai H, Wen W, et al. Adolescent and adult soy food intake and breast cancer risk: results from the Shanghai Women's Health Study. Am J Clin Nutr 2009;89:1920-6.

16. Wu AH, Ziegler RG, Horn-Ross PL, Nomura AM, West DW, Kolonel LN, et al. Tofu and risk of breast cancer in Asian-Americans. Cancer Epidemiol Biomarkers Prev 1996;5:901-6.

17. Ganai AA, Faroogi H. Bioactivity of genistein: a review of in vitro and in vivo studies. Biomed Pharmacother 2015;76:30-8.

18. Lamartiniere CA, Cotroneo MS, Fritz WA, Wang J, Mentor-Marcel R, Elgavish A. Genistein chemoprevention: timing and mechanisms of action in murine mammary and prostate. J Nutr 2002;132:552S-8S.

19. Spagnuolo C, Russo GL, Orhan IE, Habtemariam S, Daglia M, Sureda A, et al. Genistein and cancer: current status, challenges, and future directions. Adv Nutr 2015;6:408-19.

20. Cappelletti V, Fioravanti L, Miodini P, Di Fronzo G. Genistein blocks breast cancer cells in the G(2)M phase of the cell cycle. J Cell Biochem 2000;79:594-600.

21. Frey RS, Li J, Singletary KW. Effects of genistein on cell proliferation and cell cycle arrest in nonneoplastic human mammary epithelial cells: involvement of Cdc2, p21(waf/cip1), p27(kip1), and Cdc25C expression. Biochem Pharmacol 2001;61: 979-89.

22. Ornelas IM, Silva TM, Fragel-Madeira L, Ventura AL. Inhibition of PI3K/Akt pathway impairs G2/M transition of cell cycle in late developing progenitors of the avian embryo retina. PLoS One 2013;8:e53517.

23. Ou Y, Ma L, Ma L, Huang Z, Zhou W, Zhao C, et al. Overexpression of cyclin B1 antagonizes chemotherapeutic-induced apoptosis through PTEN/Akt pathway in human esophageal squamous cell carcinoma cells. Cancer Biol Ther 2013;14:45-55.

24. Stoppa-Lyonnet D, Ansquer Y, Dreyfus H, Gautier C, Gauthier-Villars M, Bourstyn E, et al. Familial invasive breast cancers: worse outcome related to BRCA1 mutations. J Clin Oncol 2000;18:4053-9.

25. Ding $\mathrm{Q}$, Gros R, Limbird LE, Chorazyczewski J, Feldman RD. Estradiol-mediated ERK phosphorylation and apoptosis in vascular smooth muscle cells requires GPR 30. Am J Physiol Cell Physiol 2009;297:C1178-87.

26. Xu X, Wagner KU, Larson D, Weaver Z, Li C, Ried T, et al. Conditional mutation of Brcal in mammary epithelial cells results in blunted ductal morphogenesis and tumour formation. Nat Genet 1999:22:37-43.

27. Ludwig T, Fisher P, Ganesan S, Efstratiadis A. Tumorigenesis in mice carrying a truncating Brcal mutation. Genes Dev 2001;15: 1188-93.

28. Prossnitz ER, Arterburn JB, Sklar LA. GPR30: a G protein-coupled receptor for estrogen. Mol Cell Endocrinol 2007;265-266:138-42.

29. Peshkin BN, Alabek ML, Isaacs C. BRCA1/2 mutations and triple negative breast cancers. Breast Dis 2010;32:25-33. 
30. Xiang T, Ohashi A, Huang Y, Pandita TK, Ludwig T, Powell SN, et al. Negative regulation of AKT activation by BRCA1. Cancer Res 2008:68:10040-4.

31. Ge C, Yu M, Zhang C. G protein-coupled receptor 30 mediates estrogen-induced proliferation of primordial germ cells via EGFR/
Akt/ß-catenin signaling pathway. Endocrinology 2012;153:3504-16 32. Privat M, Aubel C, Arnould S, Communal Y, Ferrara M, Bignon YJ. Breast cancer cell response to genistein is conditioned by BRCA1 mutations. Biochem Biophys Res Commun 2009;379:785-9. 